CESTARO, R. AJUSTE de curvas pelo método dos quadrados mínimos na elaboração de modelo matemático para estimativa da produção de soja no estado de Rondônia

\title{
AJUSTE DE CURVAS PELO MÉTODO DOS QUADRADOS MÍNIMOS NA ELABORAÇÃO DE MODELO MATEMÁTICO PARA ESTIMATIVA DA PRODUÇÃO DE SOJA NO ESTADO DE RONDÔNIA
}

\author{
Romeu Cestaro ${ }^{1}$
}

\begin{abstract}
RESUMO
Este artigo tem por objetivo abordar a utilização do ajuste de curvas pelo método dos quadrados mínimos na elaboração de um modelo matemático que seja capaz de estimar a produção de soja para a safra 2015/16 no Estado de Rondônia. Para tanto, dados referentes às safras de soja anteriores foram coletados a partir da base de dados da Companhia Nacional de Abastecimento (CONAB). Verificou-se que o modelo obtido realizou uma boa aproximação com a função tabelada, considerando-se que o maior erro relativo percentual observado tenha sido de $5,78 \%$ e erro relativo médio de $2,75 \%$; além disso, a estimativa para a produção de soja em Rondônia para a safra 2015/16, dada pelo modelo, está bastante próxima da estimativa da CONAB para o mesmo período, apresentando uma diferença percentual de $1,39 \%$.
\end{abstract}

Palavras-chave: Métodos numéricos. Método dos Quadrados mínimos. Modelagem matemática. Produção de soja. Rondônia.

\section{INTRODUÇÃO}

Uma maneira de se trabalhar com uma função definida por uma tabela de valores é a interpolação polinomial, na qual os pontos dados concernem à função interpoladora. Contudo, Ruggiero e Lopes (2010) observam que existem situações nas quais é necessário obter um valor aproximado para um ponto contido fora do intervalo de dados (quando se deseja extrapolar) ou os valores da tabela provêm de algum tipo de experimento físico ou pesquisa cujos valores podem conter erros não previsíveis, quando um método interpolador pode não ser aconselhável.

Neste contexto, define-se o objetivo primordial deste trabalho, que é abordar o ajuste de curvas pelo método dos quadrados mínimos na elaboração de um modelo matemático que seja capaz de estimar a produção de soja para a safra 2015/16 no Estado de Rondônia, representando uma boa aproximação para os valores tabelados, inerentes à produção das

\footnotetext{
1 Especializando em Tópicos Especiais em Matemática pela Universidade Cândido Mendes. Licenciatura em Matemática pela Universidade Norte do Paraná (UNOPAR) e Bacharelado em Sistemas de Informação pelo Centro Universitário Luterano de Ji-Paraná (CEULJI/ULBRA). Instrutor no Serviço Nacional de Aprendizagem Industrial (SENAI) de Ji-Paraná/RO. E-mail: romeucestaro@gmail.com.
} 
CESTARO, R. AJUSTE de curvas pelo método dos quadrados mínimos na elaboração de modelo matemático para estimativa da produção de soja no estado de Rondônia

safras de anos anteriores, permitindo a realização da estimativa (extrapolação) com uma boa margem de segurança.

Com o desenvolvimento deste trabalho, procura-se responder a algumas questões a respeito da utilização deste método: (I) O método dos quadrados mínimos é capaz de realizar um bom ajuste sobre os dados da produção de soja, resultando, assim, em um modelo matemático representativo? (II) O modelo matemático obtido é capaz de estimar a produção de soja no Estado de Rondônia para a safra 2015/2016, dentro de uma margem de erro aceitável?

Para alcançar o objetivo proposto, utilizou-se como recurso metodológico a pesquisa bibliográfica, baseada nos fundamentos apresentados por autores como Franco (2006), Ruggiero e Lopes (2010), Sperandio, Mendes e Silva (2014), e documental, a partir do levantamento dos indicadores das safras de soja do Estado de Rondônia, tendo como fonte a base de dados da Companhia Nacional de Abastecimento (CONAB) (BRASIL, 2016).

\section{AJUSTE DE CURVAS PELO MÉTODO DOS QUADRADOS MÍNIMOS - CASO DISCRETO E CASO NÃO LINEAR}

O método dos quadrados mínimos pode ser definido como um método de aproximação de funções que consiste na projeção vetorial ortogonal sobre um subespaço. Isto é, ocorre a aproximação de $f$, uma função contínua definida em um intervalo $[a, b]$, por uma combinação linear de funções, dada pela Função (1), cujos coeficientes devem ser determinados de modo que a norma euclidiana ponderada do vetor erro $\varphi-f$ resulte a menor possível, ou seja, que a distância seja mínima entre ambas as funções (FRANCO, 2006; SPERANDIO; MENDES; SILVA, 2014).

$$
\varphi(x)=\alpha_{1} g_{1}(x)+\alpha_{2} g_{2}(x)+\cdots+\alpha_{n} g_{n}(x)
$$

O ajuste de curvas pelo método dos quadrados mínimos, através do qual sabe-se que ocorre a aproximação de uma função $y=f(x) \in E$ por um polinômio de grau $m, P_{m}(x)=$ $F(x) \in E^{\prime}$, pode acontecer, conforme observa Franco (2006, p. 248), “[...] tanto no caso em que $f(x)$ é conhecida por sua expressão analítica, isto é, $f(x) \in C[a, b]$ (caso contínuo), como no caso em que $f(x)$ é dada por pares de pontos, como aqueles obtidos a partir de experimentos (caso discreto)". 


\section{FORSCIENCE}

CESTARO, R. AJUSTE de curvas pelo método dos quadrados mínimos na elaboração de modelo matemático para estimativa da produção de soja no estado de Rondônia

\subsection{Caso discreto}

Quanto ao caso discreto, suas definições e propriedades são dadas por Ruggiero e Lopes (2010). Considerados um número $\boldsymbol{m}$ de pontos tabelados $\left(x_{1}, f\left(x_{1}\right)\right),\left(x_{2}, f\left(x_{2}\right)\right), \ldots,\left(x_{m}, f\left(x_{m}\right)\right)$ e $n$ funções $g_{1}(x), g_{2}(x), \ldots, g_{n}(x)$, de alguma forma escolhidas, deve-se determinar os coeficientes $\boldsymbol{\alpha}_{1}, \boldsymbol{\alpha}_{2}, \ldots, \boldsymbol{\alpha}_{n}$, de modo que a Função (1) se aproxime ao máximo de $\boldsymbol{f}(\boldsymbol{x})$, ou seja, que resulte mínima a soma dos quadrados dos desvios. Estes coeficientes são aqueles que minimizam a Função (2):

$$
\begin{gathered}
F\left(\alpha_{1}, \alpha_{2}, \ldots, \alpha_{n}\right)=\sum_{k=1}^{m}\left[f\left(x_{k}\right)-\varphi\left(x_{k}\right)\right]^{2}=\sum_{k=1}^{m}\left[f\left(x_{k}\right)-\alpha_{1} g_{1}\left(x_{k}\right)-\right. \\
\left.\alpha_{2} g_{2}\left(x_{k}\right)-\ldots-\alpha_{n} g_{n}\left(x_{k}\right)\right]^{2}
\end{gathered}
$$

Para que seja exato o ajuste do modelo aos dados do problema trabalhado, o mínimo da referida função deve ser zero. Para que se obtenha um ponto de mínimo da Função (2), os pontos críticos $\left(\alpha_{1}, \alpha_{2}, \ldots, \alpha_{n}\right)$ devem ser encontrados, sendo necessário o cálculo das derivadas parciais, as quais deverão ser igualadas a zero. A Função (2), ao ser derivada parcialmente em relação a $\alpha_{j}$, resulta em:

$$
\begin{gathered}
\left.\frac{\delta F}{\delta \alpha_{j}}\right|_{\left(\alpha_{1}, \alpha_{2}, \ldots, \alpha_{n}\right)} \\
=2 \sum_{k=1}^{m}\left[f\left(x_{k}\right)-\alpha_{1} g_{1}\left(x_{k}\right)-\alpha_{2} g_{2}\left(x_{k}\right)-\ldots-\alpha_{n} g_{n}\left(x_{k}\right)\right]\left[-g_{j}\left(x_{k}\right)\right]
\end{gathered}
$$

Igualando-se a Função (3) a zero, obtém-se: 
CESTARO, R. AJUSTE de curvas pelo método dos quadrados mínimos na elaboração de modelo matemático para estimativa da produção de soja no estado de Rondônia

$$
\begin{gathered}
\sum_{\boldsymbol{k}=\mathbf{1}}^{\boldsymbol{m}}\left[\boldsymbol{f}\left(\boldsymbol{x}_{\boldsymbol{k}}\right)-\boldsymbol{\alpha}_{\mathbf{1}} \boldsymbol{g}_{\mathbf{1}}\left(\boldsymbol{x}_{\boldsymbol{k}}\right)-\ldots-\boldsymbol{\alpha}_{\boldsymbol{n}} \boldsymbol{g}_{\boldsymbol{n}}\left(\boldsymbol{x}_{\boldsymbol{k}}\right)\right]\left[\boldsymbol{g}_{\boldsymbol{j}}\left(\boldsymbol{x}_{\boldsymbol{k}}\right)\right]=\mathbf{0}, \boldsymbol{j}=\mathbf{1}, \ldots, \boldsymbol{n} \\
\left\{\begin{array}{l}
\sum_{k=1}^{m}\left[f\left(x_{k}\right)-\alpha_{1} g_{1}\left(x_{k}\right)-\ldots-\alpha_{n} g_{n}\left(x_{k}\right)\right] g_{1}\left(x_{k}\right)=0 \\
\sum_{k=1}^{m}\left[f\left(x_{k}\right)-\alpha_{1} g_{1}\left(x_{k}\right)-\ldots-\alpha_{n} g_{n}\left(x_{k}\right)\right] g_{2}\left(x_{k}\right)=0 \\
\sum_{k=1}^{m}\left[f\left(x_{k}\right)-\alpha_{1} g_{1}\left(x_{k}\right)-\ldots-\alpha_{n} g_{n}\left(x_{k}\right)\right] g_{n}\left(x_{k}\right)=0
\end{array}\right.
\end{gathered}
$$

Isoladas as variáveis do Sistema (4), obtém-se um sistema linear de $n$ equações e $n$ incógnitas. Trata-se de um sistema composto por equações conhecidas como equações normais:

$$
\left\{\begin{array}{l}
{\left[\sum_{k=1}^{m} g_{1}\left(x_{k}\right) g_{1}\left(x_{k}\right)\right] \alpha_{1}+\ldots+\left[\sum_{k=1}^{m} g_{n}\left(x_{k}\right) g_{1}\left(x_{k}\right)\right] \alpha_{n}=\sum_{k=1}^{m} f\left(x_{k}\right) g_{1}\left(x_{k}\right)} \\
{\left[\sum_{k=1}^{m} g_{1}\left(x_{k}\right) g_{2}\left(x_{k}\right)\right] \alpha_{1}+\ldots+\left[\sum_{k=1}^{m} g_{n}\left(x_{k}\right) g_{2}\left(x_{k}\right)\right] \alpha_{n}=\sum_{k=1}^{m} f\left(x_{k}\right) g_{2}\left(x_{k}\right)} \\
{\left[\sum_{k=1}^{m} g_{n}\left(x_{k}\right) g_{1}\left(x_{k}\right)\right] \alpha_{1}+\ldots+\left[\sum_{k=1}^{m} g_{n}\left(x_{k}\right) g_{n}\left(x_{k}\right)\right] \alpha_{n}=\sum_{k=1}^{m} f\left(x_{k}\right) g_{n}\left(x_{k}\right)}
\end{array}\right.
$$

\subsection{Caso não linear}

Além dos casos discretos, existem aqueles casos considerados não lineares, ou seja, casos de famílias de funções que, segundo Ruggiero e Lopes (2010), não apresentam linearidade nos parâmetros (no caso de uma função exponencial, por exemplo), o que requer que os dados do problema modelado sejam linearizados através de uma transformação conveniente. O método dos quadrados mínimos é, então, aplicado sobre o problema linearizado, o que pode não gerar parâmetros ótimos dentro do critério dos quadrados mínimos, por estar sendo ajustado o problema linearizado e não o problema original. 


\section{FORSCIENCE}

CESTARO, R. AJUSTE de curvas pelo método dos quadrados mínimos na elaboração de modelo matemático para estimativa da produção de soja no estado de Rondônia

\begin{tabular}{|c|c|}
\hline Modelo não linear & $\begin{array}{c}\text { Transformação } \\
\text { correspondente }\end{array}$ \\
\hline$Y=a * e^{b x}$ & $\ln Y=\ln a+b x$ \\
\hline$Y=a * b^{x}$ & $\ln Y=\ln a+(\ln b) * x$ \\
\hline$Y=a * x^{b}$ & $\ln Y=a+b x_{1}+c x_{2}$ \\
\hline$Y=l^{a+b x_{1}+c x_{2}}$ & $\frac{1}{Y}=a+b x_{1}+c x_{2}$ \\
\hline$Y=\frac{1}{a+b x_{1}+c x_{2}}$ & $\ln \left(\frac{1}{Y}-1\right)=a+b x_{1}+c x_{2}$ \\
\hline$Y=\frac{1}{1+l^{a+b x_{1}+c x_{2}}}$ &
\end{tabular}

Quadro 1- Transformações de modelos não lineares Fonte: Adaptado de Barroso et al. (1987, p. 339).

\section{PRODUÇÃO DE SOJA NO ESTADO DE RONDÔNIA}

O Estado de Rondônia, quarto maior da Região Norte do Brasil em extensão territorial, vem conquistando participação cada vez mais expressiva no quantitativo nacional da produção de soja, cuja exportação movimentou, em nível nacional, mais de 286 milhões de dólares, entre os meses de janeiro a setembro de 2015. O vegetal ocupa, atualmente, o posto de segundo produto mais exportado do Estado, representando 36,7\% das exportações rondonienses, perdendo apenas para a carne bovina (RONDÔNIA, 2015b).

O aumento sucessivo na produção de soja é observado desde o ciclo 2006/07, o último em que ocorreu redução de área no Estado, quando foram plantados 90,4 mil hectares. Destacam-se, sobretudo, os indicadores relativos às últimas safras, cujos quantitativos de produção sobressaíram-se aos demais a exemplo do desempenho obtido pela safra de 2014/15, beneficiado pelo aumento das áreas de produção de soja para novas regiões do Estado e pela manutenção dos investimentos em mecanização e adoção de novas tecnologias na lavoura. Além disso, o governo do Estado avalia que o avanço da agricultura sobre áreas de pastagem deve ser o principal fator de crescimento da área plantada com soja em Rondônia para a safra 2015/16 (RONDÔNIA, 2015a; SALOMÃO, 2015).

A Tabela 1, apresentada a seguir, relaciona o quanto se produziu em toneladas de soja no Estado de Rondônia entre as safras 2006/07 a 2014/15, com base nos dados da CONAB: 


\section{FORSCIENCE}

CESTARO, R. AJUSTE de curvas pelo método dos quadrados mínimos na elaboração de modelo matemático para estimativa da produção de soja no estado de Rondônia

Tabela 1 - Produção de soja no Estado de Rondônia

\begin{tabular}{lc}
\hline Safra & Produção (em 1.000 t) \\
\hline $2006 / 07$ & 277,5 \\
$2007 / 08$ & 311,6 \\
$2008 / 09$ & 326,5 \\
$2009 / 10$ & 384,3 \\
$2010 / 11$ & 425,3 \\
$2011 / 12$ & 462,2 \\
$2012 / 13$ & 539,3 \\
$2013 / 14$ & 607,7 \\
$2014 / 15$ & 732,9 \\
\hline
\end{tabular}

Fonte: Brasil (2016).

\section{MODELO MATEMÁTICO PARA ESTIMATIVA DA PRODUÇÃO DE SOJA NO ESTADO DE RONDÔNIA}

Tendo como base o quantitativo em toneladas de soja referente ao que o Estado de Rondônia produziu entre as safras de 2006/07 a 2014/15, pretende-se, através do ajuste de curvas pelo método dos quadrados mínimos, obter um modelo matemático representativo que possibilite a obtenção de valores não tabelados, oportunizando a projeção da produção de soja para safras futuras. Os seguintes pares ordenados constituirão a função tabelada:

$$
\begin{array}{lll}
\left(x_{1}, f\left(x_{1}\right)\right)=(1 ; 277,5) & \left(x_{2}, f\left(x_{2}\right)\right)=(2 ; 311,6) & \left(x_{3}, f\left(x_{3}\right)\right)=(3 ; 326,5) \\
\left(x_{4}, f\left(x_{4}\right)\right)=(4 ; 384,3) & \left(x_{5}, f\left(x_{5}\right)\right)=(5 ; 425,3) & \left(x_{6}, f\left(x_{6}\right)\right)=(6 ; 462,2) \\
\left(x_{7}, f\left(x_{7}\right)\right)=(7 ; 539,3) & \left(x_{8}, f\left(x_{8}\right)\right)=(8 ; 607,7) & \left(x_{9}, f\left(x_{9}\right)\right)=(9 ; 732,9)
\end{array}
$$

O objetivo é encontrar uma função que seja um bom ajuste aos dados da produção de soja, dentro do que se considere uma boa margem de segurança. Para tanto, o primeiro passo é a geração do diagrama de dispersão com os pares ordenados (6); a partir do gráfico gerado, a tendência dos dados é analisada, para que se possa escolher o modelo de função adequado para a linearização do problema em questão. 


\section{FORSCIENCE}

CESTARO, R. AJUSTE de curvas pelo método dos quadrados mínimos na elaboração de modelo matemático para estimativa da produção de soja no estado de Rondônia

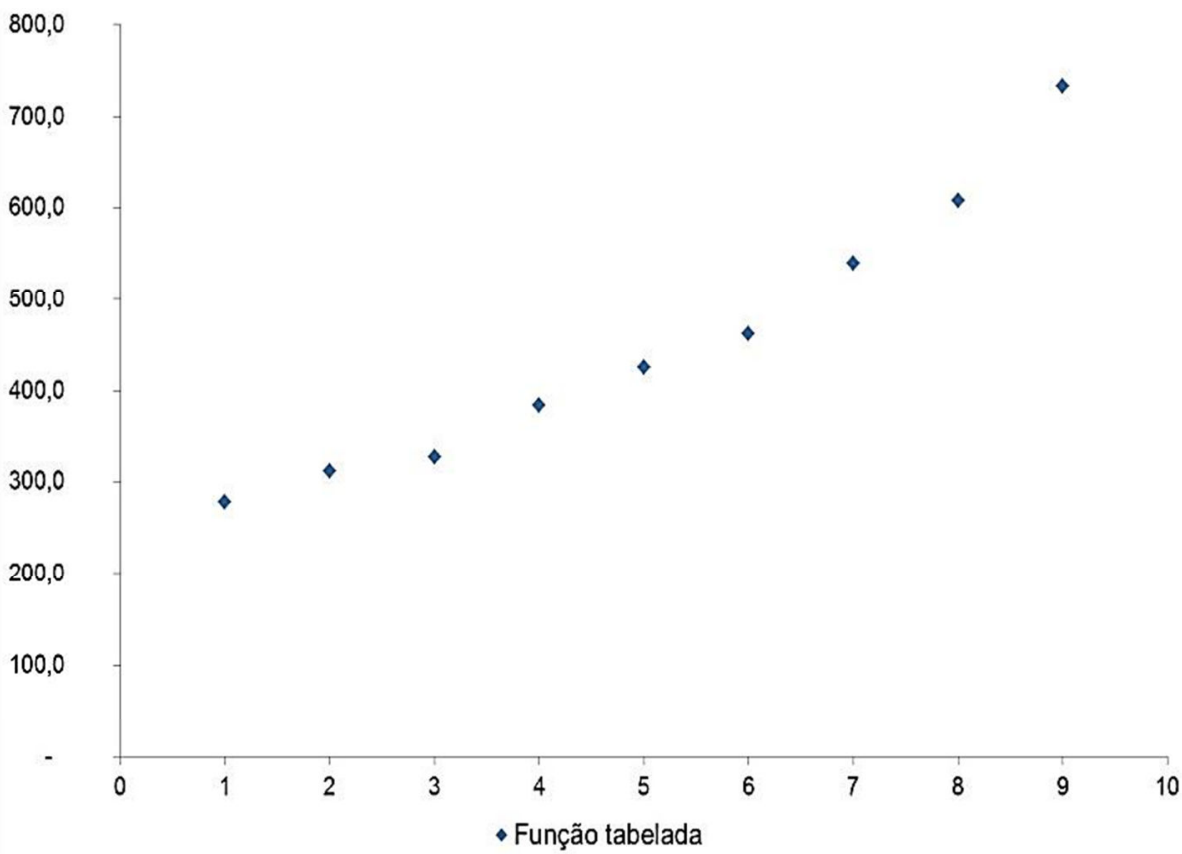

Gráfico 1 - Dispersão dos dados da função tabelada Fonte: Elaboração do autor.

A dispersão dos dados, observada no Gráfico 1, torna evidente a não linearização dos parâmetros da função tabelada, cuja disposição dos pares ordenados assemelha-se a uma curva exponencial. Sugere-se, portanto, que o ajuste da curva dos dados do problema em questão possa ser realizado a partir de:

$$
f(x) \approx \varphi(x)=\alpha_{1} e^{\alpha_{2} x}
$$

Sendo um caso não linear, o processo de ajuste pelos quadrados mínimos não se baseará em $f(x)$ diretamente. Antes disso, é requerida a linearização do problema apresentado, dado por:

$$
z=\ln (f(x)) \approx \ln \left(\alpha_{1} e^{\alpha_{2} x}\right)=\ln \left(\alpha_{1}\right)+\alpha_{2} x=\phi(x)
$$

Portanto, o ajuste por quadrados mínimos ocorrerá na Equação (8), encontrando-se $\phi=a_{1}+a_{2} x$, onde $a_{1}=\ln \left(\alpha_{1}\right)$ e $a_{2}=\alpha_{2}$. 


\section{FORSCIENCE}

CESTARO, R. AJUSTE de curvas pelo método dos quadrados mínimos na elaboração de modelo matemático para estimativa da produção de soja no estado de Rondônia

Tabela 2 - Obtenção de $z=\ln (f(x))$

\begin{tabular}{cc}
\hline $\boldsymbol{f}(\boldsymbol{x})$ & $\boldsymbol{z}=\boldsymbol{\operatorname { l n }}(\boldsymbol{f}(\boldsymbol{x}))$ \\
\hline 277,5 & 5,626 \\
311,6 & 5,742 \\
326,5 & 5,788 \\
384,3 & 5,951 \\
425,3 & 6,053 \\
462,2 & 6,136 \\
539,3 & 6,290 \\
607,7 & 6,410 \\
732,9 & 6,597 \\
\hline
\end{tabular}

Fonte: Elaboração do autor.

Definidos os valores de $z=\ln (f(x))$ (TABELA 2) e escolhidas as funções $g_{1}(x)=1$ e $g_{2}(x)=x$, deve-se solucionar as equações do Sistema (5), encontrando, desta forma, o Sistema (9):

$$
\left\{\begin{array}{c}
9 a_{1}+45 a_{2}=54,5931 \\
45 a_{1}+285 a_{2}=280,0426
\end{array}\right.
$$

Com a resolução do Sistema (9), são obtidos os resultados $a_{1}=5,4764$ e $a_{2}=$ 0,1179. Sabendo-se que $a_{1}=\ln \left(\alpha_{1}\right)$ e $a_{2}=\alpha_{2}$ e tendo sido conhecidos os valores de $a_{1}$ e $a_{2}$, têm-se:

$$
\begin{gathered}
\alpha_{1}=e^{a_{1}} \\
\boldsymbol{\alpha}_{\mathbf{1}}=\boldsymbol{e}^{\mathbf{5 , 4 7 6 4}} \\
\alpha_{1}=238,98 \\
\alpha_{2}=0,1179
\end{gathered}
$$

Considerando-se o ajuste da curva pela Função (7) e os valores conforme (10), são realizadas as devidas substituições para que se obtenha o modelo matemático pretendido para a estimativa da produção de soja:

$$
\varphi(x)=238,98 e^{0,1179 x}
$$




\section{FORSCIENCE}

CESTARO, R. AJUSTE de curvas pelo método dos quadrados mínimos na elaboração de modelo matemático para estimativa da produção de soja no estado de Rondônia

\section{RESULTADOS}

Para se avaliar a confiabilidade do modelo matemático proposto, realizou-se uma comparação entre os dados reais da produção de soja em Rondônia referente às safras 2006/07 a 2015/16 e os dados virtuais obtidos pelo modelo elaborado, como observado na Tabela 3:

Tabela 3 - Comparação entre os dados reais e os dados obtidos pelo modelo matemático

\begin{tabular}{cccc}
\hline$x$ & $f(x)$ & Modelo $\varphi(x)$ & Erro relativo (\%) \\
\hline 1 & 277,5 & 268,9 & 3,10 \\
2 & 311,6 & 302,5 & 2,91 \\
3 & 326,5 & 340,4 & 4,25 \\
4 & 384,3 & 383,0 & 0,34 \\
5 & 425,3 & 430,9 & 1,32 \\
6 & 462,2 & 484,8 & 4,89 \\
7 & 539,3 & 545,5 & 1,15 \\
8 & 607,7 & 613,7 & 0,99 \\
9 & 732,9 & 690,5 & 5,78 \\
\hline
\end{tabular}

Fonte: Elaboração do autor.

Nota: Produção expressa em mil toneladas (1.000t).

Conclui-se que o modelo matemático obtido é bastante representativo, apresentando erro relativo médio de $2,75 \%$ com relação aos indicadores da CONAB, compreendendo-se ter sido uma boa escolha para o ajuste da curva de dados o modelo de função (7). O Gráfico 2 ilustra o ajuste do modelo matemático obtido, descrito pela Função (11), aos dados da função tabelada.

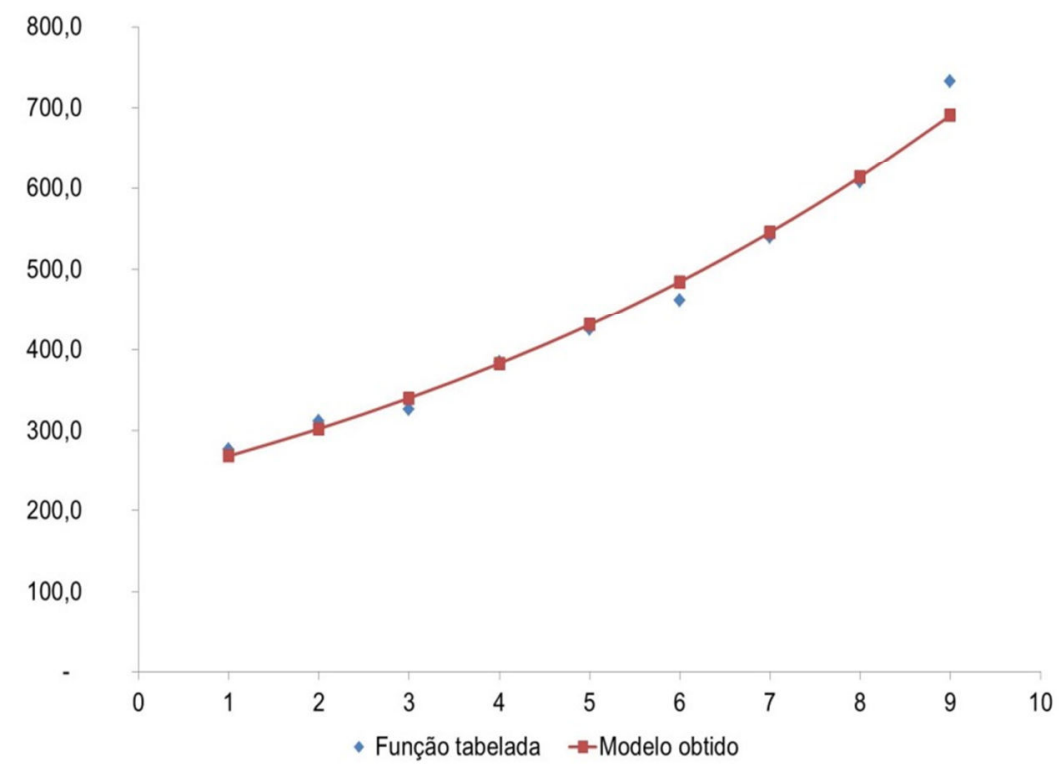

Gráfico 2 - Função tabelada versus modelo obtido Fonte: Elaboração do autor.

ForSci.: r. cient. IFMG, Formiga, v. 4, n. 1, p. 114-124, jan./jun. 2016 
CESTARO, R. AJUSTE de curvas pelo método dos quadrados mínimos na elaboração de modelo matemático para estimativa da produção de soja no estado de Rondônia

A validade do modelo matemático é também observada no caso da extrapolação dos dados, ou seja, na projeção da produção de soja para safras futuras, não tabeladas. Para a safra de 2015/16, o modelo obtido estima que a produção de soja em Rondônia chegue às 777.000 toneladas, apresentando diferença percentual de 1,39\% com relação à estimativa realizada pela CONAB no mês de junho de 2016, para a mesma safra (787.800 toneladas).

\title{
6 CONCLUSÃO
}

Pôde-se observar, por meio do presente trabalho, a validade do ajuste de curvas pelo método dos quadrados mínimos na elaboração de um modelo matemático representativo, que estime a produção de soja no Estado de Rondônia com base em quantitativos de safras anteriores.

Verificou-se que o modelo obtido realizou uma boa aproximação com os dados do problema trabalhado, considerando-se que o maior erro relativo percentual observado nos testes tenha sido de $5,78 \%$ e erro relativo médio de $2,75 \%$ com relação aos indicadores da CONAB.

Observou-se que a função obtida não gerou parâmetros necessariamente ótimos, algo que pode ser atribuído ao fato de ter sido trabalhado o problema linearizado, e não o problema original. Contudo, ficou evidente que ela é bastante representativa, especialmente quando se trata da extrapolação de dados, que é o objetivo pretendido para o referido modelo.

Aliás, é esta justamente a ideia ao se aplicar o método dos quadrados mínimos em vez de um método interpolador. Neste caso, observou-se que o modelo obteve uma estimativa de produção de soja para a safra 2015/16 bastante próxima da estimativa da CONAB para a mesma safra, apresentando diferença percentual de 1,39\%.

\section{LEAST-SQUARES BASED CURVE FITTING FOR DEVELOPING A MATHEMATICAL MODEL TO ESTIMATE SOYBEAN PRODUCTION IN RONDONIA}

\begin{abstract}
This paper intends to approach the use of least-squares based curve fitting for developing a mathematical model to estimate the soybean production for 2015/16 crop year in Rondônia. For this purpose, data on previous soybean crop years were collected from the Companhia Nacional de Abastecimento (CONAB) database. It was found that the obtained model made a good approximation to the tabulated function, considering that the greatest relative error percentage observed was $5.78 \%$, and average relative error of $2.75 \%$; also, the estimate for ForSci.: r. cient. IFMG, Formiga, v. 4, n. 1, p. 114-124, jan./jun. 2016
\end{abstract}


CESTARO, R. AJUSTE de curvas pelo método dos quadrados mínimos na elaboração de modelo matemático para estimativa da produção de soja no estado de Rondônia

soybean production in Rondonia for $2015 / 16$ crop year, given by the model, is pretty close to CONAB estimate for the same period, presenting a percentage difference of $1.39 \%$.

Keywords: Numerical methods. Method of least squares. Mathematical modeling. Soybean production. Rondônia.

\section{REFERÊNCIAS}

BARROSO, L. C. et al. Cálculo numérico: com aplicações. 2. ed. São Paulo: Harbra, 1987.

BRASIL. Companhia Nacional de Abastecimento. Séries históricas. 2016. Disponível em: $<$ http://conab.gov.br/conteudos.php?a=1252\&t=2>. Acesso em: 19 jun. 2016.

FRANCO, N. B. Cálculo numérico. São Paulo: Pearson Prentice Hall, 2006.

RONDÔNIA. Área e produção de soja crescem 20\% em Rondônia. 2015a. Disponível em: $<$ http://www.rondonia.ro.gov.br/2015/02/39656/>. Acesso em: 3 maio 2016.

Produção de soja em Rondônia tem expectativa de crescimento acima da média nacional para a próxima safra. 2015b. Disponível em: $<\mathrm{http}$ ://www.rondonia .ro.gov.br/2015/10/88536/>. Acesso em: 03 maio 2016.

SALOMÃO, R. Área da soja deve ser 10\% maior em Rondônia, estima governo. 2015. Disponível em: <http://revistagloborural.globo.com/Noticias/Agricultura/Soja/noticia/ 2015/09/area-de-soja-deve-ser-10-maior-em-rondonia-estima-governo.html>. Acesso em: 03 maio 2016.

RUGGIERO, M. A. G.; LOPES, V. L. da R. Cálculo numérico: aspectos teóricos e computacionais. 2. ed. São Paulo: Makron Books: Pearson, 2010.

SPERANDIO, D.; MENDES, J. T.; SILVA, L. H. M. Cálculo numérico. 2. ed. São Paulo: Pearson Education do Brasil, 2014.

Recebido em: 14/05/2016

Aprovado em: 20/06/2016

Publicado em: 05/07/2016 